\title{
EPISOMIC CONTROL OF MUTATION IN SALMONELLA TYPHIMURIUM
}

\author{
G. W. P. DAWSON and P. F. SMITH-KEARY \\ Deportment of Genetics, Trinity College, Dublin
}

Received I.vi.6.2

\section{INTRODUCTION}

From extensive studies of the genetic behaviour of unstable genes in maize, McClintock (1951, I953, 1955, 1956) has concluded that there exists a type of genetic unit that has no permanent location in the structure of the chromosome. When these elements are temporarily attached to a chromosomal gene they modify its phenotypic expression or enhance its rate of mutation or both. McClintock has called these units "controlling elements" and it appears that they exist in many different forms, distinguished by the loci with which they can become associated. We have studied unstable genes in Salmonella typhimurium and found that their behaviour can best be explained in similar terms. In order to avoid committing ourselves to the possible view that these genetic units in bacteria are identical with controlling elements in maize we propose to call them "controlling episomes".

\section{MATERIALS AND METHODS}

Most of the materials and methods that were used in these studies have been described in previous papers (Smith-Keary, I96o; Dawson and Smith-Keary, Ig60).

In the accounts of the experiments we have used the following abbreviations for the different media :

\footnotetext{
MM minimal medium.

EMM minimal medium enriched with broth.

$\mathrm{MM}+\mathrm{L} \quad$ minimal medium supplemented with 0.002 per cent. $d l$-leucine.

$\mathrm{MM}+\mathrm{P} \quad$ minimal medium supplemented with 0.002 per cent. $l$-proline.

E.MM-L enriched minimal medium supplemented with 0.002 per cent. $d l$-leucine.

E.MM $-\mathrm{P} \quad$ enriched minimal medium supplemented with 0.002 per cent. $l$-proline.

E.MM(arab) + LL EMM containing $0 \cdot 2$ per cent. arabinose in place of glucose and supplemented with $0 \cdot 004$ per cent. $d l$-leucine.
}

The designation of genes follows that in the previous papers. The prefix su indicates a suppressor locus. Thus su-leuA- 6 is the sixth independently isolated suppressor of leucine requirement and arose by mutation at the su-leuA locus. A

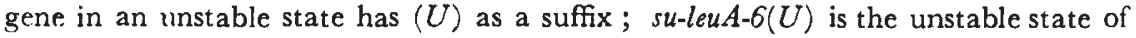
su-leu. $A-6$. 


\section{RESULTS}

(i) The reversions of leu-151

Three types of reversions of leu-I5I can be distinguished by their growth on MM:

Fast (F); large colonies on MM after 24 hours.

Slow (S); large colonies on MM after 48 hours.

Unstable slow (US); colonies on MM range from minute to large, the latter reaching a maximum size after 48 hours.

On $\mathrm{MM}+\mathrm{L}$ and EMM all three types form large colonies after 24 hours. The $\mathrm{F}$ reversions probably arise by backmutation of the leu-I5I site while the $\mathrm{S}$ reversions are known to arise by mutation at a suppressor locus (su-leuA) that is adjacent to the leu locus (SmithKeary, I96o; Dawson and Smith-Keary, I960). In this paper we will demonstrate that the US reversions probably arise by the appearance of instability at the su-leuA locus.

Independently isolated US strains can show very different degrees of instability. For example, in one experiment (Smith-Keary, 1958), two spontaneous US reversions were plated on to $\mathrm{MM}$ and after incubation for 3 days the plates showed the characteristic range of colony size. Eight colonies deriving from one reversion were plated on to $\mathrm{MM}$ and $\mathrm{MM}+\mathrm{L}$ and it was found that between 80 per cent. and 90 per cent. of the viable cells did not form colonies on MM. Twenty colonies from the other reversion were similarly plated and it was found that between 99.8 per cent. and roo per cent. of the viable cells did not form colonies on $\mathrm{MM}$. In other strains the percentage may be as low as 50. The different proportions of auxotrophic cells that colonies of different US strains contain is probably best interpreted as reflecting different degrees of instability, although there is the alternative possibility that the unreverted cells of different US strains differ in their ability to feed the auxotrophic cells. Either of these explanations is consistent with McClintock's concept of controlling elements. The first is consistent with their control of the rate of mutation; the second with their control of phenotypic expression.

In addition to arising spontaneously from $l e u-15 I$, US strains arise spontaneously in all $\mathrm{S}$ strains that are due to single site mutations within the su-leuA locus but not in su-leuA-I which is a multi-site mutant.

\section{(ii) Slows and semi-fasts derived from US strains}

$\mathrm{S}$ strains have been isolated from all the US strains studied and these are phenotypically identical to the spontaneous $S$ reversions.

The $\mathrm{S}$ and US strains also mutate to a semi-fast (SF) type, which on MM has a growth rate intermediate between that of the $F$ and $S$ strains. The SF types may be stable, or, when isolated from a US strain, they may be unstable, manifesting a similar degree of instability 
to that of the US strain from which they were isolated. Since the transduction leu-I5I $(\times)$ SF (selecting for leucine independence on E.MM) yields only $\mathrm{S}$ transductants, the $\mathrm{SF}$ types are probably due to the mutation of an unlinked modifier of su-leuA.

The S, US and SF strains have never been observed to mutate directly to wild type.

(iii) Auxotrophs derived from US strains

Two series of experiments were carried out to determine whether or not the auxotrophs derived from US strains (secondary auxotrophs) are identical to the original su-leuA+leu-I5I strain (primary auxotroph). Firstly the reversion spectra of the primary and secondary auxotrophs were compared, and secondly, a secondary auxotroph was used in transductions with leu-I5I.

TABLE I

Frequencies of spontaneous reversion (per $10^{8}$ bacteria plated) of leu- $\mathrm{I}^{\mathrm{j}} \mathrm{I}_{0}$ to the $F$, $S$ and US types in 15 independent experiments. $1 \cdot 0-3 \cdot 0 \times 10^{8}$ bacteria per plate

\begin{tabular}{|c|c|c|c|c|c|c|c|c|c|}
\hline \multirow{2}{*}{$\begin{array}{l}\text { Total bacteria } \\
\text { plated } \times 10^{-8}\end{array}$} & \multirow{2}{*}{$\begin{array}{l}\text { Reversions } \\
\text { observed }\end{array}$} & \multicolumn{4}{|c|}{ Reversions tested } & \multicolumn{4}{|c|}{ Frequencies } \\
\hline & & total & $\mathrm{F}$ & $\mathrm{S}$ & US & total & $\mathbf{F}$ & $\mathrm{S}$ & US \\
\hline 9.00 & $5^{2}$ & 20 & I & I 4 & 5 & $5 \cdot 72$ & 0.29 & $4 \cdot 00$ & I $\cdot 43$ \\
\hline $7 \cdot 00$ & 42 & 35 & I & 10 & 24 & $6 \cdot 00$ & 0.17 & $J \cdot 71$ & $4 \cdot 12$ \\
\hline 2700 & $4^{I}$ & 20 & I & 7 & I 2 & $I \cdot 5^{2}$ & $0 \cdot 08$ & 0.53 & 0.91 \\
\hline 30.00 & 20 & 20 & 7 & I & 12 & $6 \cdot 66$ & $2 \cdot 33$ & 0.33 & 4.00 \\
\hline $39^{\circ} 00$ & $5^{6}$ & 20 & 2 & 2 & I 6 & $I \cdot 43$ & $0 \cdot 14$ & 0.14 & I 15 \\
\hline $9 \cdot 06$ & $3 \mathrm{I}$ & 20 & o & 6 & I 4 & $3 \cdot 42$ & 0.00 & $\mathrm{I} \cdot \mathrm{O} 3$ & $2 \cdot 39$ \\
\hline $10 \cdot 3$ & 22 & 20 & 0 & 7 & I 3 & $2 \cdot 14$ & 0.00 & 0.75 & I 39 \\
\hline $0 \cdot 96$ & 41 & 28 & 2 & 23 & 3 & $42 \cdot 71$ & 3.05 & $35 \cdot 08$ & $4 \cdot 5^{8}$ \\
\hline $10 \cdot 2$ & $5^{2}$ & 20 & I & I 6 & 3 & $5 \cdot 10$ & 0.26 & $4 \cdot 08$ & 0.76 \\
\hline $6 \cdot 3$ & 108 & 60 & $5^{6}$ & 4 & o & $17 \cdot 14$ & $16 \cdot 00$ & $I \cdot 14$ & 0.00 \\
\hline $8 \cdot 4$ & 60 & 40 & 0 & 40 & o & $7 \cdot 14$ & 0.00 & $7 \cdot 14$ & 0.00 \\
\hline $9 \cdot 0$ & I 23 & 40 & 25 & I 5 & 0 & I 3.67 & $8 \cdot 54$ & $5 \cdot 13$ & 0.00 \\
\hline $3 \cdot 16$ & 54 & 20 & 0 & 20 & o & 17.09 & 0.00 & 17.09 & 0.00 \\
\hline $5 \cdot 95$ & 32 & 29 & 10 & 19 & o & $5 \cdot 37$ & $1 \cdot 58$ & $3 \cdot 5^{2}$ & 0.00 \\
\hline $6 \cdot 85$ & 21 & $40^{*}$ & 0 & 39 & I & 3.07 & 0.00 & $3 \cdot 00$ & 0.07 \\
\hline & & means & & & & $9 \cdot 2 !$ & $2 \cdot 18$ & $5 \cdot 64$ & I. 39 \\
\hline
\end{tabular}

* Nineteen colonies obtained from additional control plates.

Six secondary auxotrophs (designated leu-I $I_{S} S_{S 1}$ to $l e u-I 5^{I} S_{6}$ ) were isolated independently from US strains and the frequency of reversion of each estimated by plating washed samples, each of $2 \% \mathrm{IO}^{8}$ bacteria, on EMM or MM. After 4 days incubation samples of the reversions were characterised for leucine requirement. The results of these experiments (table 2) can be compared with the results of similar cxperiments using the primary auxotroph (table I). leu-I $I_{S} I_{S 1}$ and leu- $I_{5} I_{S 6}$ reverted to the $\mathrm{F}, \mathrm{S}$ and US types at frequencies not significantly different from those obtained in experiments using the primary auxotroph, while leu-I5IS2, $3,4,5$ reverted to the $\mathrm{S}$ and/or US types at very 
much higher frequencies. In one experiment using leu-I5IS the washed suspension was plated both on MM and EMM; the very small number of reversions recovered on $\mathrm{MM}$ indicates that the majority of the reversions on the EMM plates are due to many mutational events,

TABLE 2

Frequencies of reversion (per $10^{8}$ bacteria plated) of leu-1 $5 \mathrm{I}$ s strains to the $F, S$ and US types $0.5-2.0 \times 10^{8}$ bacteria per plate

\begin{tabular}{|c|c|c|c|c|c|c|c|c|c|c|}
\hline \multirow{2}{*}{ Strain } & \multirow{2}{*}{ Medium } & \multirow{2}{*}{$\begin{array}{l}\text { Total } \\
\text { bacteria } \\
\text { plated } \\
\times 10^{\cdots 8}\end{array}$} & \multirow{2}{*}{$\begin{array}{l}\text { Revisions } \\
\text { observed }\end{array}$} & \multicolumn{4}{|c|}{ Reversions tested } & \multicolumn{3}{|c|}{ Frequencies } \\
\hline & & & & total & $\mathbf{F}$ & $\mathbf{S}$ & US & F & S & US \\
\hline$S_{I}$ & EMM & $\mathrm{II} \cdot 5$ & 76 & 19 & 2 & I 6 & I & 0.69 & $5 \cdot 56$ & 0.34 \\
\hline $\mathrm{S}_{2}$ & EMM & $\begin{array}{r}3 \\
3.22\end{array}$ & 4000 & 20 & 0 & 0 & 20 & 0 & 0 & $1240^{34}$ \\
\hline$S_{2}$ & EMM & $1 \cdot 12$ & 24 large & 10 & 10 & 0 & 0 & $21 \cdot 43$ & 0 & 12500 \\
\hline & MM & & $\begin{array}{l}\text { 1400o small } \\
\text { I3 large }\end{array}$ & $\begin{array}{l}20 \\
10\end{array}$ & $\begin{array}{r}0 \\
10\end{array}$ & $\begin{array}{l}\circ \\
\circ\end{array}$ & $\begin{array}{r}20 \\
0\end{array}$ & - & - & \\
\hline$S_{3}$ & EMM & c. $1 \cdot 2$ & & 18 & 0 & 5 & 13 & 0 & $37 \cdot 27$ & $96 \cdot 89$ \\
\hline$S_{4}$ & EMM & c. $1 \cdot 2$ & 196 & 19 & 1 & 6 & 12 & $8 \cdot 59$ & $5 \mathrm{I} \cdot 54$ & 103.08 \\
\hline$S_{5}^{4}$ & EMM & c. $1 \cdot 2$ & $8000+$ & 22 & 0 & 22 & 0 & 0 & $6666+$ & 0 \\
\hline 56 & EMM & c. I. & $5^{6}$ & 22 & 5 & 9 & 8 & $10 \cdot 6 x$ & 19.09 & ז 6.97 \\
\hline
\end{tabular}

Mean frequency of $\mathrm{F}$ reversions (per $10^{8}$ bacteria plated) $=5 \cdot 90$.

occurring either very late in the growth of the culture or on the enriched plates (or both). Thus the instability has been retained at this locus even though it has mutated from su-leuA- to su-leu $A^{+}$.

In these experiments the mean frequency of $\mathrm{F}$ reversions, 4.12 per $10^{8}$ bacteria plated, was not greatly in excess of the corresponding frequency $(2 \cdot 18)$ obtained from similar experiments using the primary auxotroph.

TABLE 3

Mean number of colonies per $10^{8}$ bacteria plated in transductions involving leu-15I and leu- $\mathrm{I}_{5} \mathrm{I}_{\mathrm{S}}$

\begin{tabular}{|l|r|r|}
\hline \multirow{2}{*}{ Recipient } & \multicolumn{2}{|c|}{ Donor } \\
\cline { 2 - 3 } & leu-15I & leu-I5ISI \\
\hline leu-I5I & $10 \cdot 84$ & $6 \cdot 00$ \\
leu-I5ISI & $2 \cdot 35$ & $\mathrm{x} \cdot 22$ \\
\hline
\end{tabular}

Table 3 shows the results when leu-I5IS1 was used in transduction experiments with leu-I5I, selecting for leucine independence on EMM. The mean number of colonies per $10^{8}$ bacteria plated in the transductions $l e u-I_{5 I} I_{S 1}(\times) l e u-I 5^{I}$ and $l e u-I 5 I(\times) l e u-I 5 I_{S 1}$ are not significantly greater than in the controls using homologous phage and we conclude that $l e u-I 5 I$ and $l e u-I_{5} I_{S 1}$ are identical alleles. 


\section{(iv) Transductions using US strains}

The genetic factors responsible for the US phenotype can be transduced into leu-I5I (and into any leu or $\operatorname{araB}$ mutant), and table 6 sets out the results of 5 independent experiments using different US donors isolated directly from leu-I5I. Phage raised on each of the US strains was used to infect leu-I5I and leucine independent transductants selected by plating on EMM. In all experiments the majority of the transductants were unstable slow growing, although with donors $\mathrm{US}(\mathrm{I}), \mathrm{US}(2), \mathrm{US}(4)$ and $\mathrm{US}(5)$ a few $\mathrm{S}$ transductants were also recovered. In preparing the phage, care was taken to ensure that the populations of US bacteria used as donors contained a high proportion of US bacteria (c. 50 per cent.) and a low proportion of S and SF bacteria $(<0.5$ per cent.). Consequently, it is reasonably certain that the $\mathrm{S}$ colonies were not due to the presence of $\mathrm{S}$ bacteria in the donor population, but rather that the stable su-leuA alleles have been transduced from the US bacteria. This, together with the evidence that the maximum growth rate of the US type is similar to the growth rate of the $\mathrm{S}$ type, and that both can mutate to the SF type (which has never been detected to arise from a strain other than S or US) indicates that both the US and S bacteria have the constitution su-leuA leu-I5I, and that the cause of the instability is closely linked to su-leuA.

\section{(v) Extent of the instability}

Since instability can be detected at all known sites within su-leuA, we can now ask whether in any particular strain the instability is confined to a particular site within su-leuA or whether the acquisition or loss of instability at a particular site affects the stability at other sites.

If the auxotroph su-leuA-x+(U) leu-I5I is isolated from a su-leuA-x(U) leu-I5I strain any S or US reversion of this auxotroph will be su-leuA-x leu-I5I if the instability is confined to the $x$ site in su-leuA; on the other hand, if the instability extends over all or part of su-leuA the S and US reversions will be su-leuA-x leu-I5I, su-leuA-y leu-I5I, su-leuA-z leu-I5I . . . etc. where $x, y, z, \ldots$ etc. represent different sites within su-leuA and it should be possible to detect recombination between these su-leuA mutants (Dawson and Smith-Keary, I96o).

To distinguish between these alternatives, a US strain was isolated

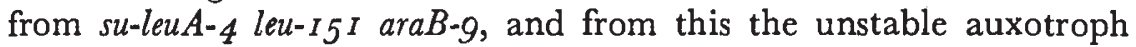
su-leu $A-4^{+}(U)$ leu-I I I araB-g obtained. Five colonies of this auxotroph were inoculated into broth, grown overnight, plated on $\mathbf{M M}$ and incubated. From each series of plates a $\mathrm{S}$ reversion was selected. We wished to know whether all these $\mathrm{S}$ reversions were mutant at site 4 or at different sites in the su-leuA locus. The procedure was as follows.

The $a r a B-9$ marker was removed from each of the 5 strains and from the original su-leuA-4 leu-I5I araB-9 strain by transduction using 
$\mathrm{H}_{4}$ phage. Phage $\mathrm{PLT}_{22}$ grown on each of the five strains $\left(\mathrm{araB}^{+}\right)$ was used to infect the original su-leuA-4 (araB-9) and phage grown on the original su-leuA-4 $\left(a r a B^{+}\right)$used to infect each of the five strains $(a r a B-9) . a r a B^{+}$transductants were selected by plating on EMM(arab) $+\mathrm{LL}$ and at least 80 colonies from each transduction characterised

Starting with :-

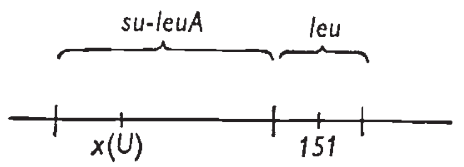

unstable slow growing

Select from unstable slow growing :-

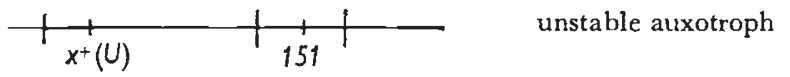

From unstable auxotroph select slow growing reversions. These will be :-

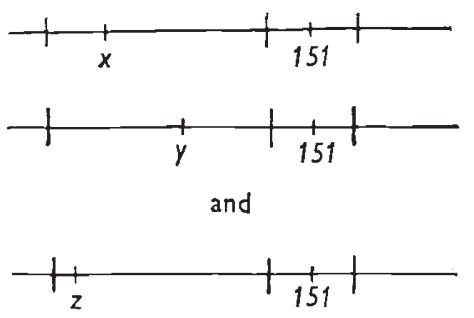

if instability confined to $x$ site all $\mathrm{S}$ reversions: will be su-leuA-x leu-I5I

or

if instability affects other sites within su-leuA $\mathrm{S}$ reversions will be su-leuA-y leu-I5I, su-leuA-z leu-I5I etc.

if the second of these alternatives occurs it can be detected by the following transduction :-

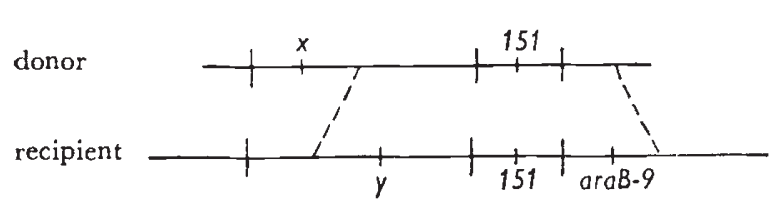

su-leuA-x and su-leuA-y can recombine with the original su-leuA-x allele to restore su-leu $A^{+}$. Thus in the trans. duction su-leuA-y leu-15I $\operatorname{araB}-g(X)$ su-leuA-x leu-I5I (selecting for $a r a B^{+}$transductants) some leucine auxotrophs will be recovered.

Frg. 1.-Method used for detecting whether or not the instability is confined to a particular site within su-leuA (for description see text).

for leucine requirement. If any of the reversions is mutant at a su-leu $\mathrm{A}$ site other than su-leuA-4, then from the argument and results presented in a previous paper (Dawson and Smith-Keary, 1960), leucine auxotrophs should be recovered in one of the reciprocal transductions involving that mutant and su-leuA-4. Since no auxotrophs were recovered in any of the transductions, the mutant sites in the five strains must be either identical or very closely linked to the su-leuA-4 site. We conclude that in a particular unstable strain the instability is probably confined to a particular su-leuA site and is retained at that site through successive back and forward mutations. 


\section{(vi) Episomes as the cause of instability at su-leuA}

The similarities between the instability patterns observed in these experiments and in maize, where instability is known to be caused by the presence of controlling elements (McClintock, I95 I, I953, I955, 1956), suggested to us that similar elements may be responsible for the instability at su-leuA in Salmonella typhimurium and, as a working hypothesis we propose that instability appears at su-leuA when such an clement (which we call a controlling episome) becomes attached to a su-leuA site. This hypothesis will fully account for the results of the preceeding experiments. It is also consistent with the observation that over the 4 years that we have used leu-15I, the frequency of spontaneous US reversions has decreased, and in the existing stock (leu-I5Ie) US reversions are rare. This is apparent from the results set out in table 1 , where mutational experiments carried out in $195^{8}$ and 1959 are listed chronologically. Similarly, existing stocks of su-leuA-3 leu-15I, su-leuA-4 leu-I $I_{I}$ and su-leuA-6 leu-I5I have lost their ability to produce US colonies spontaneously.

This can be accounted for if we assume that the original stocks of leu-I 5I, su-leuA-3leu-I 5I, su-leuA-4leu-I 5 I and su-leuA-6 leu-I 5 I contained controlling episomes which have either been lost or have become more or less stably integrated at some unknown site and are thus not now readily available for attachment to su-leuA.

In order to demonstrate the similarity between controlling elements and controlling episomes we must show that controlling episomes can be transposed between su-leuA and an unknown location, or even better, between su-leuA and a known locus. This demonstration is provided by the experiments described in the next section.

\section{(vii) Transposition of controlling episomes}

The approach to this problem was suggested by the observations stated above, which lead to the prediction that the existing strain of leu-I5I has a low frequency of readily available controlling episomes, and from the observation that when a stable su-leuA- allele, together with leu-15I, is introduced into an $\operatorname{araB}-9$ strain by transduction many of the slow growing transductants are unstable, thus suggesting that araB.9 strains have controlling episomes which are available for attachment to $s u$-leuA. If this is so we also expect the stable su-leuA ${ }^{+}$ allele to gain instability when it is introduced with $l e u-15 I$ into $a r a B-9$ and it is this situation which we have most fully investigated.

Phage $\mathrm{H}_{4}$ was used to effect the transduction $\operatorname{araB}-g(\times)$ leu-I5Ie, selecting on EMM(arab) +LL, and from among the ara $B^{+}$transductants I I strains (leu-I5I A to $\mathrm{K}$ ) were isolated which were phage sensitive and leucine requiring. Each strain was grown in broth, washed, diluted and $10^{8}$ bacteria spread on each of $8 \mathrm{EMM}$ plates. After 4 days' incubation every reversion was characterised by subculturing on to $\mathrm{MM}$ and $\mathrm{MM}+\mathrm{L}$. As a control, 15 single colony isolates of 
leu-15I were similarly grown, plated and the reversions characterised. To ensure that the results were comparable, care was taken that equivalent numbers of bacteria were spread on each plate; this was achieved by diluting each washed suspension to an optical density corresponding to $10^{\theta}$ bacteria per $\mathrm{ml}$. and plating $0 \cdot 1 \mathrm{ml}$. samples. By this method the number of viable bacteria plated was kept within the limits $1 \pm 0 \cdot 15 \times 10^{8}$.

The results (tables 4 and 5) demonstrate that when leu-15I is introduced into the $a r a B-9$ genome the frequency of US reversions is

TABLE 4

Frequencies of reversion of leu- $5^{\mathrm{I}_{B}}$ to the $F, S$ and US types. $8 \times 10^{8}$ bacteria from each culture plated on EMM (I $10^{8}$ bacteria per plate)

\begin{tabular}{|c|c|c|c|c|}
\hline \multirow{2}{*}{ Culture } & \multicolumn{4}{|c|}{ Reversions observed } \\
\hline & Total & F & $\mathrm{s}$ & US \\
\hline I & 23 & 4 & I 6 & 3 \\
\hline 2 & 14 & 2 & 10 & 2 \\
\hline 3 & $\mathrm{I}$ & 0 & I & 0 \\
\hline 4 & I0 & 2 & 6 & 2 \\
\hline 5 & 2 & 0 & 2 & 0 \\
\hline $\begin{array}{l}3 \\
6\end{array}$ & 12 & I & II & 0 \\
\hline 7 & 18 & I & I5 & 2 \\
\hline 8 & 17 & 3 & 14 & 0 \\
\hline 9 & Io & 3 & 6 & I \\
\hline IO & 20 & 4 & I 6 & 0 \\
\hline I I & 12 & 2 & IO & 0 \\
\hline I2 & 6 & 0 & 2 & 4 \\
\hline I3 & 4 & 2 & 0 & 2 \\
\hline$I_{4}$ & 3 & 0 & 3 & 0 \\
\hline I 5 & 9 & I & 8 & 0 \\
\hline Total & I6I & 25 & 120 & I 6 \\
\hline $\begin{array}{c}\text { Reversions per } 10^{8} \\
\text { bacteria plated }\end{array}$ & I 34 & 0.21 & $I \cdot 00$ & 0.13 \\
\hline
\end{tabular}

over 32 times as great as in the control experiments using leu-I5Ie. There are also small increases in the frequency of $\mathrm{F}$ and $\mathrm{S}$ reversions, but these are primarily due to the high frequencies of reversion in cultures $\mathrm{E}$ and $\mathrm{H}$ (to $\mathrm{F}$ type) and in cultures $\mathrm{C}$ and $\mathrm{I}$ (to $\mathrm{S}$ type) and are probably attributable to mutations occurring during the early growth of these cultures. When leu-I5I is in the araB-g genome the frequency of US reversions is also much higher than the corresponding frequency in early experiments with the original leu-15I strain (leu-I5Io) (I 39 per $10^{8}$ bacteria plated, table $\mathrm{I}$ ).

To demonstrate that the instability was not due to the different genetic backgrounds provided by the leu-I5 $I_{e}$ and araB-9 strains, phage PLT22 grown on 5 US and $3 \mathrm{~S}$ reversions of leu-15I (araB-9 genome) was used to infect leu-15 $I_{\mathrm{e}}$ and the leucine independent transductant characterised by subculturing on to MM. 
The results (table 6) show that with $\mathrm{S}$ donors only $\mathrm{S}$ transductants were recovered and that with US donors the majority of the transductants were US. Since the instability at su-leuA is maintained when

TABLE 5

Frequencies of reversion of leu- $\mathrm{I}_{5} \mathrm{I}$ to the $F, S$ and $U S$ types after introduction into the araB-9 genome. $8 \times 10^{8}$ bacteria of each strain plated on EMM ( $10^{8}$ bacteria per plate)

\begin{tabular}{|c|c|c|c|c|}
\hline & \multicolumn{4}{|c|}{ Reversions observed } \\
\cline { 2 - 5 } Strain & Total & F & S & US \\
& & & & \\
& & & 15 & 28 \\
A & 43 & 0 & 16 & 14 \\
C & 32 & 2 & 35 & 44 \\
D & 26 & 9 & 8 & 17 \\
E & 135 & 19 & 4 & 112 \\
F & 18 & 6 & 8 & 4 \\
G & 73 & 0 & 16 & 57 \\
H & 81 & 26 & 9 & 46 \\
I & 46 & 0 & 32 & 14 \\
J & 36 & 2 & 18 & 16 \\
K & 23 & 2 & 4 & 17 \\
\hline Totals & 601 & 67 & 165 & 369 \\
Reversions per 10 & 6.83 & $0 \cdot 76$ & 1.88 & $4 \cdot 19$ \\
bacteria plated & & & & \\
\hline
\end{tabular}

TABLE 6

Transductions using leu- $\mathrm{I}^{\mathrm{I}} \mathrm{e}$ as recipient and $S$ and US reversions of leu- $15 \mathrm{I}$ (araB-9 genonie) as donors. Selection on EMM, leucine independent transductants characterised for their ability to grow in the absence of leucine

\begin{tabular}{|c|c|c|c|}
\hline \multirow{2}{*}{$\begin{array}{c}\text { Leucine independent } \\
\text { donor }\end{array}$} & \multicolumn{2}{|c|}{ Leucine independent reversions characterised } \\
\cline { 2 - 4 } & Total & $\mathrm{S}$ & US \\
\hline S (1) & 60 & 60 & \\
S (2) & 60 & 60 & 0 \\
S (3) & 60 & 60 & 0 \\
US (1) & 60 & 4 & 56 \\
US (2) & 60 & 6 & 54 \\
US (3) & 60 & 0 & 60 \\
US (4) & 60 & 7 & 53 \\
US (5) & 60 & 2 & 58 \\
(recipient only) & 0 & 0 & 0 \\
\hline
\end{tabular}

the su-leuA(U) alleles are reintroduced into leu-I5I, the difference in mutability is not an effect of genetic background. We interpret these results as demonstrating the transposition of a controlling episome from an unknown location to a site within the leu-ara linkage group (probably within su-leuA) whilst su-leu $A^{+}$is in the araB-g genome. 
We have further been able to show the transposition of instability between su-leuA and a known locus. The experiments, in which we used the double mutant leu-I5I pro-4OI isolated from leu-I5Ie by penicillin screening, were as follows:-

I. Six broth cultures of leu-I5I pro-4OI were washed and about $10^{8}$ bacteria plated on each of $3 \mathrm{EMM}+\mathrm{P}$ and $3 \mathrm{EMM}+\mathrm{L}$ plates. After 5 days' incubation the proline independent reversions were characterised by subculturing on to $\mathrm{MM}+\mathrm{L}$. These were of 5 types (i) stable fast growing, (ii) stable slow growing, (iii) unstable slow growing, (iv) stable very slow growing, and (v) unstable very slow growing. These data (table 7) provide an estimate of the frequencies of reversion to

TABLE 7

$3 \times 10^{8}$ bacteria of 6 independent cultures of leu-151 $\mathrm{e}$ pro-401 plated on $E M M+L\left(10^{8}\right.$ bacteria per plate) and all the reversions characterised for their ability to grow in the absence of proline

\begin{tabular}{|c|c|c|c|c|c|c|}
\hline \multirow{2}{*}{ Culture } & \multicolumn{6}{|c|}{ Proline independent reversions } \\
\hline & Total & $\underset{\text { stable }}{\text { Fast }}$ & $\begin{array}{l}\text { Slow } \\
\text { stable }\end{array}$ & $\begin{array}{c}\text { Slow } \\
\text { unstable }\end{array}$ & $\begin{array}{l}\text { Very slow } \\
\text { stable }\end{array}$ & $\begin{array}{l}\text { Very slow } \\
\text { unstable }\end{array}$ \\
\hline $\begin{array}{l}\text { A } \\
\text { B } \\
\mathbf{C} \\
\text { D } \\
\mathbf{E} \\
\mathbf{F}\end{array}$ & $\begin{array}{l}38 \\
37 \\
37 \\
16 \\
45 \\
3^{8}\end{array}$ & $\begin{array}{r}25 \\
29 \\
65 \\
6 \\
8 \\
7\end{array}$ & $\begin{array}{r}12 \\
4 \\
7 \\
6 \\
12 \\
13\end{array}$ & $\begin{array}{l}0 \\
1 \\
0 \\
0 \\
2 \\
0\end{array}$ & $\begin{array}{r}1 \\
2 \\
0 \\
4 \\
20 \\
14\end{array}$ & $\begin{array}{l}0 \\
1 \\
0 \\
0 \\
3 \\
4\end{array}$ \\
\hline Total & 246 & 140 & 54 & 3 & $4^{1}$ & 8 \\
\hline $\begin{array}{c}\text { Means } \\
\text { (per culture) }\end{array}$ & $4^{t \cdot 00}$ & $23 \cdot 67$ & 9.00 & 0.50 & $6 \cdot 85$ & $1 \cdot 33$ \\
\hline
\end{tabular}

each of the proline independent types in the stable double mutant leu-15I. pro-4OI.

2. The leucine independent reversions were similarly characterised on $\mathrm{MM}+\mathrm{P}$ and $3 \mathrm{US}$ colonies which also required proline selected for further study. For brevity, these 3 strains, having the general genotype su-leuA-x $(U)$ leu-I5I pro-40I are designated leuU $(A)$ pro, leuU $(B)$ pro and $l e u U(C)$ pro.

3. Each of the leuU pro strains was grown in broth, washed and plated ( $10^{8}$ bacteria per plate) on 3 EMM $+\mathrm{L}$ plates. After 5 days' incubation the reversions on each series of plates were scored and characterised by simultaneously subculturing onto $\mathrm{MM}+\mathrm{L}$ and $\mathrm{MM}+\mathrm{P}$. Thus each reversion was characterised for $(a)$ type of proline independence and $(b)$ whether su-leuA was stable or unstable (table 8).

Comparing the frequencies of proline independent reversions of leuU pro with leu-r $5 I_{e}$ pro it is clear that while there is no increase in the 
frequency of fast reversions and no significant increase in the frequency of stable slow reversions $(P>0 \cdot I)$, there are very marked increases in the frequencies of unstable slow, stable very slow and unstable very slow reversions. Furthermore, there is a conspicuous association between instability for proline independence and stability at su-leuA and vice versa; there is a highly significant difference between the frequencies of $\mathrm{S}$ and US (leucine independent) colonies among both the proline independent stable and unstable slow reversions $\left\langle\chi_{[1]}^{2}\right.$ $=33.4 \mathrm{P}<0.00 \mathrm{r})$, and the proline independent stable and unstable very slow reversions $\left(\chi_{[2]}^{2}=31^{\circ} 4 \mathrm{P}<0 \cdot 00 \mathrm{I}\right)$.

\section{TABLE 8}

$3 \times 10^{8}$ bacteria of each leuU pro- 401 strain plated on $E M M+L\left(10^{8}\right.$ bacteria per plate $)$ and all the reversions characterised for their ability to grow in the absence of proline and in the absence of leucine

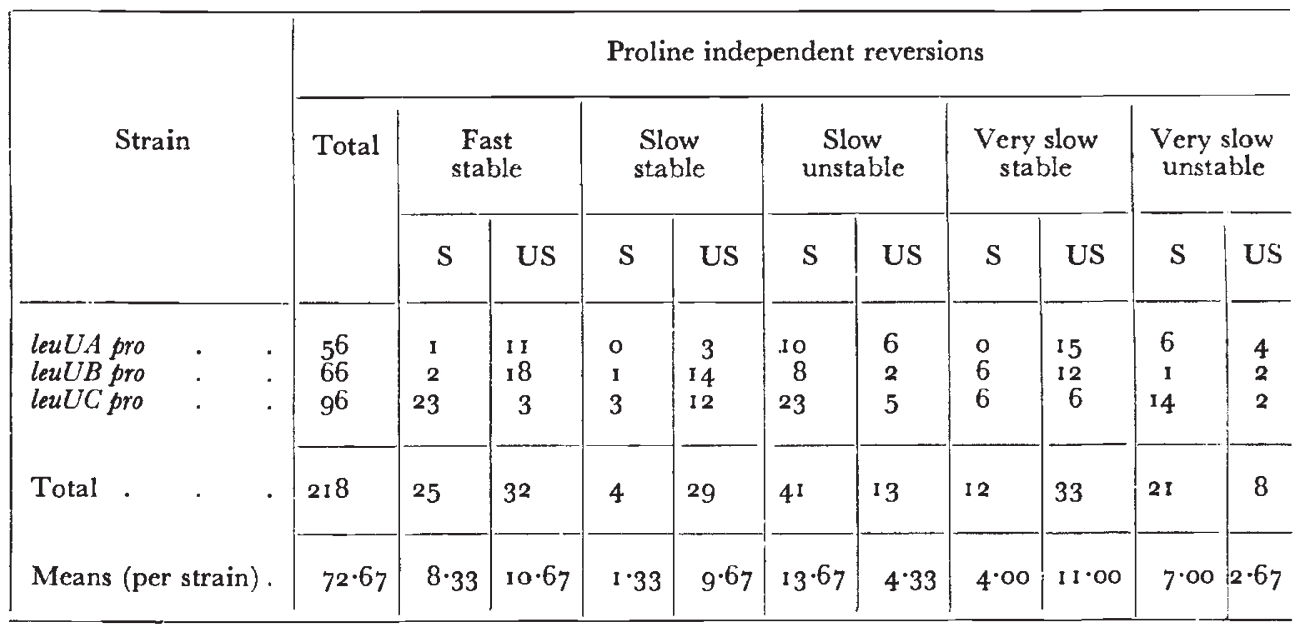

$\mathrm{S}=$ leucine independent stable slow growing.

US = leucine independent unstable slow growing.

According to our hypothesis we interpret these results as follows. In leu-I5I pro-4OI controlling episomes are not readily available and by selecting a leucine independent unstable slow growing reversion we have selected the descendants of a bacterium which contained at least one available controlling episome. Provided that this controlling episome replicites in harmony with the bacterial genome, all the bacteria in this US clone will likewise carry a detectable controlling cpisome, now located within the leu-ara linkage group. If this controlling episome can affect the proline loci (or suppressors thereof) there will be a greater chance of transposition to proline suppressors occurring in this US strain than in the stable leucine auxotroph.

Since our hypothesis attributes instability to the attachment of a controlling episome at or near the locus manifesting instability, the appearance of instability at a suppressor of proline requirement should 
be accompanied by a loss of instability from su-leuA. The results, while in very good agreement with this prediction, do show a discrepancy, 13.04 per cent. of the proline independent unstable slow and unstable very slow reversions also manifesting instability at su-leuA. This probably indicates that some bacteria contain more than one controlling episome available to these loci. Two further important points follow from the correlation of instability at su-leuA with stability at suppressors of proline requirement and vice versa. Firstly, the controlling episome must, on transposition from su-leuA, have a very strong affinity for the proline suppressor locus since, if it could transpose to any locus in the genome, it is most unlikely that any significant correlation would be detected, and secondly the low frequency of types unstable at both loci makes it unlikely that the average frequency of available controlling episomes is much greater than one per bacterium.

Strong support for our interpretation of these results in terms of controlling episomes come from the fact that the unstable suppressors of proline requirement can be introduced into leu-15I. pro-4OI by transduction, the instability being retained in the majority of the proline independent transductants. Thus instability originally associated with the su-leuA locus and transducible with it has become instability at a locus affecting proline requirement and is now transducible with this locus.

\section{DISCUSSION}

The main experimental results that have to be discussed are as follows:

I. The following diagram summarises the pattern of mutation that has been observed at the su-leuA locus;
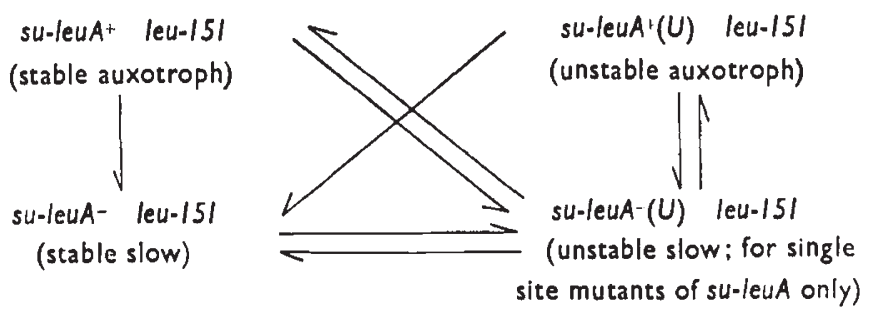

2. In mutations of su-leuA- leu-I5I to su-leuA-(U) leu-I5I to su-leuA+(U) leu-I5I and back to su-leuA-leu-I5I the instability remains associated with a particular site within su-leuA.

3. Instability cannot be detected in existing stocks of su-leuA-3 leu-I5I, su-leuA-4 leu-I5I and su-leuA-6 leu-I5I although when first isolated (1958) these strains showed considerable instability. Similarly the original leu-I5I strain has become progressively stabilised. 
4. The introduction of a stable su-leuA- allele, together with leu- $I_{j} I$, into an $a r a B-g$ results in the su-leu $A$ locus acquiring instability. Likewise, su-leu $A^{+}$acquires instability more readily in an araB-g genome than in the original or existing leu-I5I genome.

5. Unstable su-leuA- alleles can be transduced without loss of instability.

6. In su-leuA-(U) leu-I5I pro-4OI the unstable reversions for proline requirement are associated with stability at su-leuA, while the corresponding stable reversions retain the instability at su-leuA. When this apparent transfer of instability from su-leuA to loci modifying proline requirement has taken place, it can be transduced with these loci.

To account for these results we have proposed a hypothesis in terms of controlling episomes. Before discussing this in more detail we will briefly consider three other hypotheses which might be thought to account for the instability.

\section{(i) Mutator hypothesis}

Mutator genes are known in Salmonella (Miyake, 1960) and in Escherichia coli (Treffers et al., 1954; Skaar, 1956). They affect all or most of the genes with which they have been tested. If a mutator gene were responsible for the pattern of instability at the su-leuA locus it would have to possess the following special properties:

(a) It must be able to switch from an active to an inactive form, and the reverse, to explain spontaneous changes of su-leuA to and from the unstable state.

(b) It must affect some loci (su-leuA) and not others (leu) and be able to direct its activity to specific sites within the loci it affects.

(c) It must be able to switch its activity from one locus to another (from su-leuA to loci affecting proline requirement).

(d) It must affect the rates of both forward and back mutation.

This amount of special pleading makes it unprofitable to explain instability at su-leuA in terms of mutator genes that have been invoked to account for other studies. Further, the fixed location of mutator genes is incompatible with the observation that su-leuA can "pick up" instability from the $a r a B-9$ genome and with the observation that instability at su-leuA, and transducible with su-leuA, can be transferred to loci affecting proline requirement and is then transducible with these loci. Although an explanation of instability at su-leuA in terms of mutator genes is excluded it is possible that further study of mutator genes will reveal that they are parts of systems of controlling episomes; the $D t-a_{1}$ system in maize was explained in terms of $D t$ as a mutator gene until further study showed it to be a controlling element system (Rhoades, 1936, 1938; Nuffer, 1955, I961). 
(ii) Inherent instability hypothesis

This hypothesis proposes that the instability is an inherent property of the su-leuA alleles. While it will account for the fact that instability can be transduced it is excluded by the evidence for the transposition of instability from one locus to another.

\section{(iii) Aneuploidy for a chromosome fragment}

This hypothesis proposes that a US strain is aneuploid for a chromosome fragment carrying the su-leuA locus, and that a reversion to auxotrophy occurs whenever such a fragment is lost. Since the instability can be transduced together with $l e u$ and $a r a B^{+}$the fragment must also include these loci and the US strain will be:

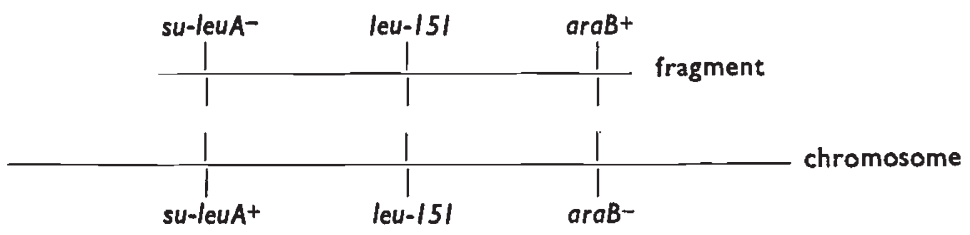

We consider this hypothesis unlikely for the following reasons:

(a) Transducing phage grown on such a US donor would be of two types, some incorporating the su-leuA- leu-I5I araB ${ }^{+}$ alleles of the fragment and others the su-leuA $A^{+} l e u-I 5^{I} a r a B^{+}$ alleles of the chromosome. When used to infect an araB-9 strain (selecting for $\mathrm{araB}^{+}$and considering only those transductants that are not $\left.l e u^{+} a r a B^{+}\right)$the former will yield almost exclusively slow growing transductants while the latter will yield only leu-I5I auxotrophs, these two classes of transductants occurring with approximately equal frequency. This is not in agreement with our observation that slow growing transductants are 27 times more frequent than leu-I5I auxotroph transductants.

(b) When a fragment is lost the resulting auxotroph should be stable; however, we have shown that auxotrophs derived from unstable slow growing strains are themselves unstable and have a much higher frequency of mutation at su-leuA than the primary auxotroph.

(c) It cannot account for the transposition of instability.

The data can best be explained in terms of controlling episomes which induce mutations. There is no evidence that the controlling episomes suppress the phenotypic expression of the genes to which they are attached; that we find instability of both su-leuA- and su-leuA+ is evidence that the attachment of the episome does not interfere with the 
phenotypic expression of the gene. The main features of this explanation are:

(a) Instability at su-leuA arises by the attachment of a controlling episome at a su-leuA site; loss of the controlling episome results in stability being regained (see fig. 2).

(b) Instability is confined to the site at which the controlling episome is attached.

(c) The presence of a controlling episome induces both forward and back mutations.

(d) Controlling episomes may be transferred from one chromosomal location to another.

While these features can account for most of the data some development is necessary to explain the different degrees of instability observed among US strains isolated from the same $\mathrm{S}$ strain, the relatively high frequency of stable slows among the transductants from leu-I $5 I(X)$ US and the progressive loss of instability from some strains during subculturing. The first may be due either to differences in the episomic content of the genetic background which affects the activity of the controlling episome at su-leuA or to different states of the controlling episome. The second demonstrates that the controlling episome is

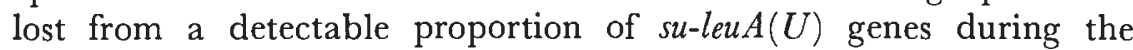
process of transduction. The loss of instability during subculturing may be explained by the transposition of the controlling episome to other loci from which transposition back to su-leuA rarely occurs. Alternatively a controlling episome may occasionally exist free instead of integrated with the chromosome; chance inclusion in daughter cells at cell division would result in some lines of cells with no controlling episomes and strains derived from these lines would be stable.

The similarities between our controlling episomes and McClintock's controlling elements are striking. Both are genetic units which show transposition from one location on the chromosome to another. Both enhance the rate of mutation of the genes to which they are attached. In maize two types of mutation have been observed at loci having an attached controlling element (I) stable mutations accompanied by loss of the element from the affected locus and (2) mutations not accompanied by loss of the element, resulting in new unstable alleles; both these types of mutation have been observed in the present study. In maize the controlling elements may function independently or they may form a two-element system in which one element activates the element located at the affected locus; we have no evidence for supposing that the controlling episomes at su-leuA are part of a two-element system. In addition to causing mutations, controlling elements may modify the expression of the gene to which they are attached; the controlling episomes at su-leuA do not appear to modify the expression of either su-leuA or of su-leuA $A^{+}$. The effects of some, but not all, controlling elements spread along the chromosome to nearby genes; the effect 
of a controlling episome at su-leuA not only does not spread to neighbouring genes but is confined to a particular site within the gene.

By using the term controlling episome we not only avoid committing ourselves to the identity of these units with controlling elements but draw attention to the fact that they are in many ways similar to those units in bacteria that are known as episomes (Jacob and Wollman, 1958). Episomes include the temperate bacteriophages, the $F$ factor in Escherichia coli and the colicinogenic factors in the Enterobacteriacee and have been the subject of recent reviews (Jacob, Schaeffer and Wollman, 1960; Sneath, 1962; Smith and Stocker, 1962). Their characteristic feature is that they are genetic particles that can exist either free in the cytoplasm or integrated with the structure of the chromosome. In the former they replicate independently of the chromosome; in the latter they replicate pari passu with the chromosome. The existence of the controlling episome as a unit integrated with the chromosome is adequately demonstrated by its high frequency of transduction with the affected locus. Its transposition from one locus to another demonstrates that it can temporarily have an autonomous existence although the present study provides no evidence that it can replicate in this state. Evidence that supports our comparison of the transposition state with the autonomous state of episomes is provided by our more recent studies which show that controlling episomes in this state, but not in the integrated state, can be partially eliminated with acridine orange. This will be described fully in a later paper but briefly we have been able to show that the frequency of unstable slows that arise in su-leuA $A^{+} l e u-15 I$ (araB-g genome)-presumably by transposition of controlling episomes to su-leuA sites where they induce mutations-is reduced by acridine orange while the frequency of stable slows that arise in an unstable slow strain-presumably by loss of a controlling episome from su-leuA-cannot be increased by acridine orange. This result is to be compared with that of Hirota (1960) who has shown that while $F$ can be eliminated by acridine orange from $F^{+}$ strains of $E$. coli (where $F$ is autonomous) it cannot be similarly eliminated from $\mathrm{Hfr}$ strains (where $F$ is integrated). Other similar instances of the elimination of episomes by treatment with acridine dyes are cited by Sneath (1962). Colicinogenic, $F$ and multiple drug resistance factors in the autonomous state are readily transmitted from one bacterium to another following cell pairing, which they initiate. In the integrated state $F$ (i.e. Hfr) and colicinogenic factors can initiate cell pairing and subsequent transfer and integration of the chromosome. We have no evidence that controlling episomes have these properties. Cell pairing is not, however, a necessary consequence of the presence of episomes as temperate bacteriophages do not have this effect.

Variant $F$ factors are known in $E$. coli which have a preference for attaching to a particular chromosomal site (Adelberg and Burns, 
1960; Richter, 1961). The high correlation between loss of a controlling episome from su-leuA and acquisition of a controlling episome by the loci affecting proline requirement, and the very high frequency with which unstables arise in some su-leuA leu-I5I strains, suggests that controlling episomes exhibit a similar preferential affinity. The preferential affinity of the $F$ factor is thought to arise by recombination occuring between the chromosome and the $F$ factor when the latter is integrated at the site for which $F$ has an affinity, and a similar explanation could account for the behaviour of the controlling episomes.

The similarity between controlling episomes and episomes and between controlling episomes and controlling elements suggests that we have here a general class of genetic unit which has no fixed location in the structure of the chromosome, although showing varying degrees of preference for particular loci, and which can exist in a free state although showing varying degrees of autonomy and ability to replicate in this state. When they have a high degree of autonomy they can greatly modify the phenotype of the cell. When they exist in an integrated state they may in addition modify the expression of the gene to which they are attached and its rate of mutation. It may be that this class of genetic unit consists of autonomous units, wholly or partly DNA (Marmur et al., I96r) the sequence of nucleotides in the whole or part of this DNA determining this pairing with specific regions of the chromosome.

This study has been primarily an investigation of instability at a particular locus in Salmonella. While we have compared our results with similar, but more extensive, studies in maize there are many other organisms in which instability has been recorded. For example, in Drosophila Demerec (1926, 1929a, 1929b) has described strains of $D$. Virilis carrying mutable genes which can mutate to each other or to wild type; in these strains selection can be made for increased instability. In $D$. pseudoobscura Race B. Mampell (1943, 1945, 1946) has shown the existence of a dominant mutator gene, located on chromosome II, which, when a Y chromosome is present induces mutations at a wide range of loci; this mutator gene is apparently infectious. Among the many species of angiosperms in which unstable genes have been described are Delphinium ajad (Demerec, I93I; Dawson, 1955), Portulaca (Blakeslee, 1920; Fabergé and Beale, 1942), Pharbitis (Imai, I934; Tabuchi, I935a, I935b; Imai and Tabuchi, I938), Pelargonium (Imai, 1936), Gossypium (Harland, 1937) and Lathyru (Imai and linuma, 1938). Amonr micro-organisms, Newcombe (1953) has described a complex pattern of radiation induced instabilities in Streptomyces, which affect colony morphology and colour; some of the mutations are stable, others are unstable and mutate to further variant types, and Newcombe has suggested that controlling elements may be responsible for this instability. Similar instabilities in Penicillium chrysogenum have also been ascribed to the presence of a controlling element (Morpugo and Sermonti, 1959). Goldstein and Smoot (I955) 
have described a strain of Escherichia coli which is characterised by having a very high rate of mutation to auxotrophy. This strain was further studied by Zamenhof (Zamenhof et al., I958a) who has shown that the instability may disappear and reappear. These, and other

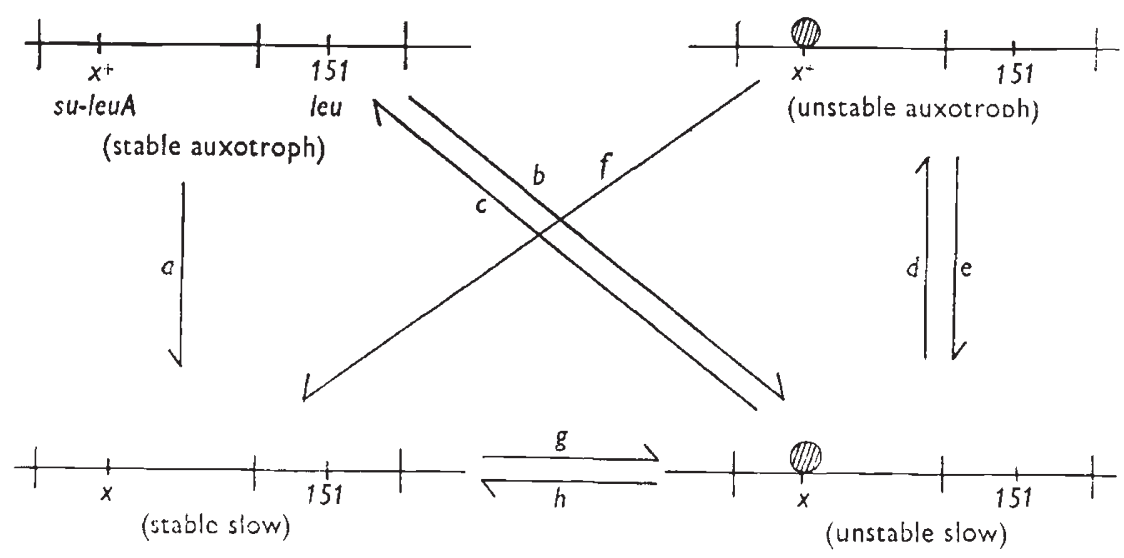

FIG. 2.-Explanation of the mutations observed at su-leuA. represents a controlling episome which, when it attaches to a site within su-leuA, causes instability.

examples of genes which can exist in stable and unstable states (Zamenhof, I945; Bunting, 1946; Lederberg, I952; Zamenhof et al., I958b) are difficult to explain in terms of Mendelian genes and suggest that controlling elements are of widespread occurrence.

\section{SUMMARY}

Unstable reversions at the su-leuA locus in strain leu-I5I of Salmonella typhimurium have been studied. This instability has been explained in terms of the movement to and from the locus of units similar to controlling elements. We have called these controlling episomes.

I. The explanation of the pattern of those mutations that we have studied is as follows and is summarised in fig. 2.

(a) Mutation of su-leuA-x+ to su-leuA-x.

(b) Mutation of su-leuA-x+ to su-leuA-x after attachment of a controlling episome.

(c) Mutation of su-leuA-x to su-leuA- $x^{+}$followed by loss of a controlling episome.

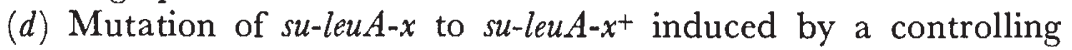
episome.

(e) Mutation of su-leuA-x+ to su-leuA-x induced by a controlling episome.

(f) Mutation of su-leuA-x+ to su-leuA-x followed by loss of controlling episome.

(g) Attachment of a controlling episome to $s u-l e u A-x$.

(h) Loss of controlling episome from su-leuA-x. 
By comparison with $a, b$, and $g$ the mutations $c, d, e, f$ and $h$ (those induced by an attached controlling episome and/or loss of the controlling episome) occur with a high frequency and always at the site of su-leuA to which the controlling episome is attached.

2. Different strains have been shown to contain different frequencies of controlling episomes available for transposition to su-leuA.

3. The transposition of controlling episomes from su-leuA to loci modifying proline requirement has been demonstrated.

4. Controlling episomes can be transduced together with the loci to which they are attached.

Acknowledgment.-The research reported herein has been sponsored by the Office, Chief of Research and Development, U.S. Department of the Army, through its European Research Office.

\section{REFERENCES}

ADELBERG, E. A., AND BURNS, S. N. 1960. Genetic variation in the sex factor of Escherichia coli. 7. Bacteriol., 79, 321-330.

BUNTING, M. I. 1946. The inheritance of color in bacteria, with special reference to Serratia marcescens. Cold Spring Harbor Symp. Quant. Biol., II, 25-32.

Blakeslee, A. F. 1920. A dwarf mutation in Portulaca showing vegetative reversions. Genetics, 5, 419-433.

Dawson, G. W. P. 1955. The inheritance of variegated flower colour in Delphinium ajacis. Heredity, 9, 409-41 2 .

DAWson, G. W. P., AND SMith-KeARy, P. F. 1960. Analysis of the su-leuA locus in Salmonella typhimurium. Heredity, 15, 339-350.

DEMEREC, M. 1926. Miniature $a-$ a second frequently mutating character in Drosophila virilis. P.N.A.S., $13,249-253$.

DEMEREC, M. 1929a. Genetic factors stimulating mutability of the miniature-gamma wing character of Drosophila virilis. P.N.A.S., ${ }_{15}, 834-838$.

DEMEREC, M. 1929b. Changes in the rate of mutability of the mutable miniature gene of Drosophila virilis. P.N.A.S., ${ }_{15}, 870-876$.

Demerec, M. I931. Behaviour of two mutable genes in Delphinium ajacis. 7. Genet., 24, 179-193.

FAbergé, A. C., AND beAle, G. H. 1942. An unstable gene in Portulaca; mutation rate at different temperatures. F. Genet., 43, 1 73-187.

Goldstern, A., AND sMoot, J. S. 1955. A strain of Escherichia coli with an unusually high rate of auxotrophic mutation. 7. Bacteriol., 70, 588-595.

HARLAND, s. c. 1937. The genetics of cotton. XVII. Increased mutability of a gene in $G$. purpurascens as a consequence of hybridisation with $G$. hirsutum. 7. Genet., 34, 1 53-1 68.

HIRoTA, Y. 1960. The effect of acridine dyes on mating type factors in $E$. coli. P.N.A.S., $46,57-64$.

Imal, x. 1934. On the mutable genes of Pharbitis, with special reference to their bearing on the mechanism of bud variation. Jour. Coll. Agric. (Tokyo), 12, 479-523.

IMAI, y. 1936. Geno- and plasmotypes of variegated Pelargoniums. F. Genet., 33, 169-1 95 .

IMAI, y., AND IINUMA, y. 1938. Variation in the flaked lines of Lathyrus coloratus. 7. Genet., 35, 42 I-430.

IMAI, Y., AND TABUCHI, K. 1938. Recurrent mutation in the flaked alleles of Pharbitis purpurea. 7. Genet., 35, 433-446.

JACOB, F., AND WOLlman, E. L. 1958. Les episomes, éleménts génétique ajoutés. C.R. Acad. Sci., 247, 154-1 56. 
JACOB, F., SCHAEFFER, P., AND WOLLMAN, E. L. 196o. Episomic elements in bacteria. In Microbial Genetics. Symp. Soc. Gen. Microbiol., 10, 67-91.

LEDERBERG, E. M. 1952. Allelic relationships and reverse mutation in Escherichia coli. Genetics, 37, 469-483.

mampelt, к. 1943. High mutation frequency in Drosophila pseudoobscura, Race B. P.N.A.S., 29, 137-144.

MAMPELX, K. I945. Analysis of a mutator. Genetics, 30, 496-505.

MAMPELL, K. 1946. Genic and non-genic transmission of mutator activity. Genetics, 3I, 589-597.

MARMUR, J., ROWND, R., FALKOW, S., BARON, L. S., SCHILDKRAUT, C., AND DOTY, P. 1961. The nature of intergenic episomal infection. P.N.A.S., 47, 972-979.

McCLINTOCK, B. 195I. Chromosome organisation and genic expression. Cold Spring Harbor Symp. Quant. Biol., 16, $13-47$.

McCLINTOCK, B. 1953. Induction of instability at selected loci in maize. Genetics, 38, 579-599.

MeCLINTOCK, B. 1955. Intranuclear systems controlling gene action and mutation. Brookhaven Symp. Biol., 8, 58-71.

Mc CLINTock, B. 1956. Controlling elements and the gene. Cold Spring Harbor Symp. Quant. Biol., 21, 197-216.

MTYAKE, т. 1960. Mutator factor in Salmonella typhimurium. Nature. $183,1586$.

MORPUGO, G., AND SERMONTI, G. 1959. Chemically-induced instabilities in a heterozygous diploid of Penicillium chrysogenum. Genetics, 44, 137-1 52.

NEWCOMBE, н. в. 1953. Radiation induced instabilities in Streptomyces. 7. Gen. Microbiol., 9, 30-36.

NUFFER, M. G. 1955. Dosage effect of multiple $D t$ loci on mutation of $a$ in the maize endosperm. Science, I2I, 399-400.

NUfFer, M. G. 1961. Mutation studies at the $A_{1}$ locus in maize. I. A mutable allele controlled by $D t$. Genetics, $46,625-640$

RHOADES, M. M. 1936. The effect of varying gene dosage on aleurone color in maize. 7. Genet., 33, 347-359.

RHOADES, M. M. 1938. Effect of the $D t$ gene on the mutability of the $a$ allele in maize. Genetics, 23, 377-397.

RICHTER, A. 1961. Attachment of wild type $F$ factor to a specific chromosomal region in a variant strain of Escherichia coli $-\mathrm{K}_{12}$; the phenomenon of episomic alteration. Genet. Res. Camb. (rg6I), 2, 333-345.

SIRKS, M. J. 1950. Sobre los genes "inestables". Genetica Iberica, 2, rog-111.

SKAAR, P. D. 1956. A binary mutability system in Escherichia coli. P.N.A.S., 42, 245-249.

SMrth, S. M., AND STOCKER, B. A. D. 1962. Colicinogeny and recombination. Brit. med. Bull., 18, 46-51.

SMITH-KEARY, P. F. 1958. An unstable mutation in Salmonella typhimurium. Nature, 181,1672 .

SMITH-KEARY, P. F. 1960. A suppressor of leucineless in Salmonella typhimurium. Heredity, $14,6 \mathrm{I}-7 \mathrm{I}$.

SNEATH, P. H. A. 1962. Sex factors as episomes. Brit. med. Bull., 18, $4^{\mathrm{r}-45}$.

TABUCH, $x$. 1935a. The mutable behaviour of Delicate genes in the Japanese morning glory. Jour. Coll. Agric. (Tokyo), 13, 373-395.

TABUCHI, $\mathrm{k}$. 1935b. A study on the mutability of the willow leaf in the Japanese morning glory. Jour. Coll. Agric. (Tokyo), 13, 415-430.

TREFFERS, H. P., SPINELLI, V. AND BELSER, N. D. 1954. A factor (or mutator gene) influencing mutation rate in Escherichia coli. P.N.A.S., 40, 1064-1071.

ZAMENHOF, s. 1945. Studies of factors influencing mutability. Experiments with unstable genes. 7. Genet., 47, 64-68.

ZAMENHOF, S., GREER, S., AND DE GIOVANNI, R. 1958a. Studies of spontaneous gene unstabilisation in Escherichia coli. 7. Bacterol., 75, 510-513.

ZAMENHOF, S., DE GIOVANNI, R., AND GREER, s. 1958b. Induced gene unstabilisation. Nature, $181,827-829$. 